\title{
APICOECTOMY TREATMENT OF AN IMPACTED MAXILLARY CANINE THAT RESISTED ORTHODONTICALIY FORCED ERUPTION
}

\author{
Constantinus Politis ${ }^{1 a^{*}}$, Jimoh Olubanwo Agbaje ${ }^{1 b}$, Yi Sun ${ }^{1 c}$, Harry Stamatakis ${ }^{2 d}$, \\ Luc Daems ${ }^{3 e}$, Ivo Lambrichts ${ }^{4 f}$
}

${ }^{1} \mathrm{KU}$ Leuven Department of Imaging and Pathology, Department of Oral and Maxillofacial Surgery,

University Hospitals Leuven, Belgium

${ }^{2}$ DFaculty of Dentistry, Department of Orthodontics, Groningen, The Netherlands

${ }^{3}$ Department of Oral and Maxillofacial Surgery, Middelheim Ziekenhuis ZNA, Antwerp, Belgium

${ }^{4}$ Faculty of Medicine, Hasselt University, Diepenbeek, Belgium

aMD, DDS, MHA, MM, PhD, Professor and Head of Department OMFS

${ }^{\circ} B D S$, DMD, FMCDS, MMI, PhD, Post doctoral fellow

'MSC, PhD, Post doctoral fellow

${ }^{d} D D S$, resident doctor orthodontics

$M D$, DDS, Professor and Senior Lecturer

'DDS, PhD, Professor and Senior Lecturer

Received: September 7,2015 Received in revised form: December 7,2015

Accepted: December 10, 2015

Cite this article:

Published online: February 09, 2016

Politis C, Agbaje JO, Sun Y, Stamatakis H, Daems L, Lambrichts I. Apicoectomy treatment of an impacted maxillary canine that resisted orthodontically forced eruption. Stoma Edu J.2016;3(1-2):22-27.

https://doi.org/10.25241/stomaeduj.2016.3(1-2).art.3

Aim: This case study describes a palatally impacted maxillary canine that was successfully brought into occlusion after initial resistance to orthodontically-induced forces.

Summary: Clinical and radiographic examinations of the impacted canine revealed a dilaceration of the apical portion of the root, which was bent, and hooked into the dense cortical bone of the nasal cavity floor. Ankylosis was excluded as the main cause of immobility. Finally, the canine was endodontically treated and an apicoectomy was performed to remove the bent tip.

Results: During the follow-up period, orthodontic force was applied to encourage canine movement. Fourteen months after the operation, the canine had descended to a functional occluding position. Twenty-six months after the operation, no signs of apical lesion or root resorption were observed. The dentition and occlusion remained stable.

Key learning point: Apical dilaceration through the cortical bone may cause immobility of an impacted canine. Apicoectomy of the bent tip following endodontic treatment of the tooth led to successful exposure and eruption of the canine, with a favorable prognosis.

Keywords: apicoectomy, impacted tooth, canine guidance.

\section{Introduction}

Maxillary canine impaction occurs with a reported prevalence of $0.8-3.3 \%$, as opposed to impacted mandibular canines, which occur less frequently. ${ }^{1-4}$ Specifically, impacted canines with palatal displacements occur at a ratio of $1: 3$ compared to those with labial displacements. ${ }^{5}$ Several etiologies have been identified that may potentially lead to impaction of a canine. For example, canine impaction may be due to failed resorption of the deciduous tooth root; early loss of the deciduous teeth, followed by lack of space in the arch; dislocation of the impacted canine and an abnormal eruption path; blockage of the eruption, due to the presence of a pathological entity in close proximity to the tooth (e.g., cysts, odontomas or supernumerary teeth); dental crowding; root dilacerations; or even failure of the eruption mechanism. Also, an eruption may be obstructed by mucosa thickening after trauma or extraction. ${ }^{5}$ It is important to identify the cause of impaction before treatment, to ensure the proper counteractive measure is included in a suitable treatment plan. Although lack of sufficient space is the most frequent etiologic factor for impaction of a maxillary canine, it has been found that palatally impacted canines are most often associated with sufficient space in the arch. ${ }^{6}$

Therefore, another cause or combination of causes should also be considered, when determining the etiology. The present report presents a case of a maxillary impacted canine that resisted an orthodontically forced eruption. Here, the treatment sequence is described, with a 1-year postoperative follow-up. 


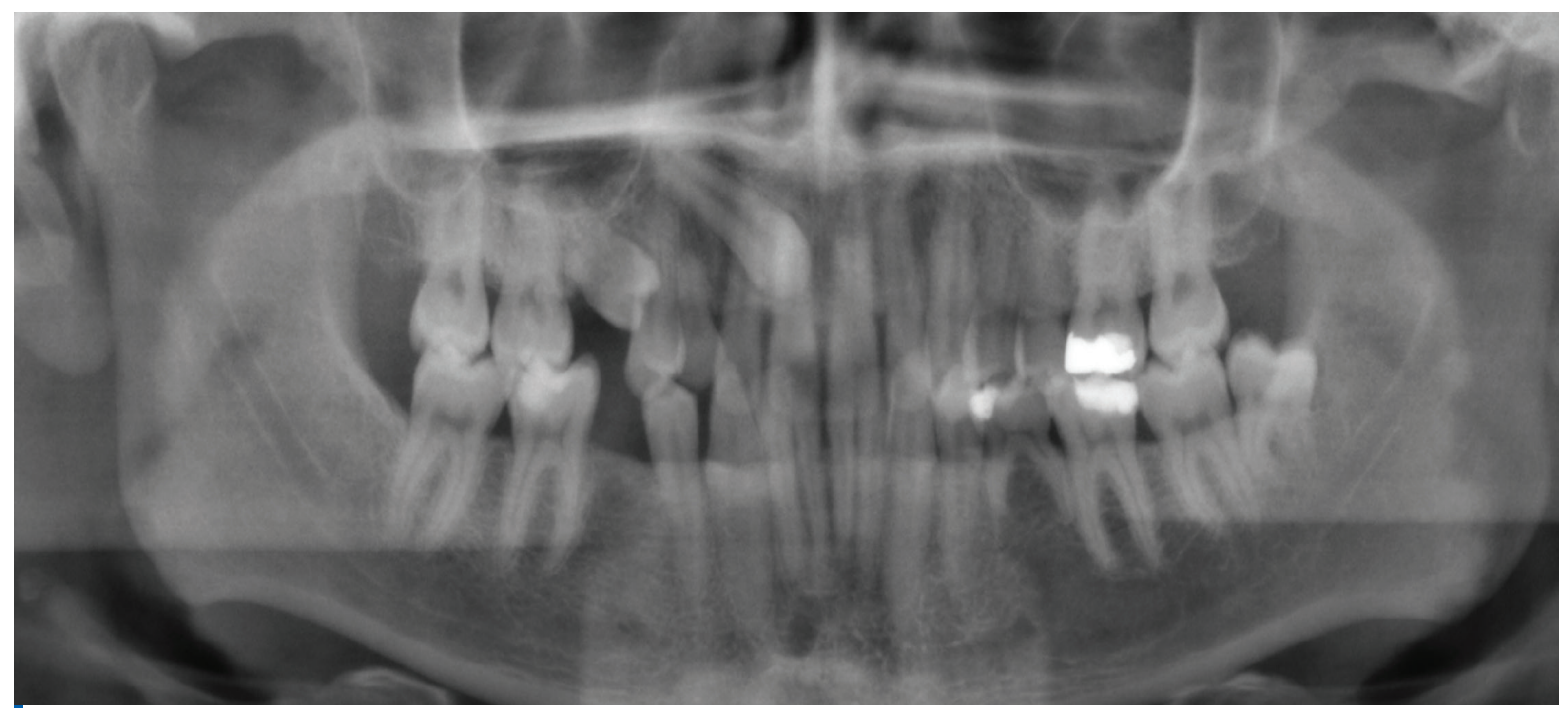

Figure 1. Panoramic radiograph of the impacted right upper canine at its initial location

\section{Case Report}

A 26-year-old male was referred to the polyclinic for dental treatment. The clinical investigation revealed agenesis of the lower second premolars with residual space at location 45 and a retained tooth 75. Additionally, impactions were present at positions 15 and 13, with residual space at position 15 and a retained tooth 53, respectively (Fig. 1). The dental arches were not crowded; spaces between the teeth were observed in both the upper and lower jaws.

The suggested starting orthodontic treatment was to place a fixed appliance in the upper jaw to open the pathways for normal eruptions of teeth 15 and 13. We decided to treat the edentulous space at position 45 with an implant at a later date. The patient's consent was received, and the treatment plan commenced.

After 6 months, tooth 38 was extracted, due to recurrent pericoronitis, and tooth 53 was removed in the same session. The linguallylocated, impacted canine (tooth 13) was exposed with a high degree of complexity, due to its deep impaction behind the root of tooth 12. A bracket was placed on the disto-palatal surface of tooth 13 . Twelve months after the canine was exposed surgically, its downward movement had halted, despite the well-prepared exposure, the firm attachment of the bracket, the sufficient space, and the favorable eruption orientation.

The canine was tested for ankylosis with the PerioTest Classic (MedizinTechnik Gulden e.K, Modautal, Germany). The test result indicated normal mobility, with no suspicion of ankylosis. A radiographic examination with panoramic radiography (Orthophos XG Plus, Sirona, Germany) revealed that the canine was bent in the apical region. The apex was curved towards the cortical bone that forms the floor of the nasal cavity, and it anchored the canine in an impacted position. Further examination with cone beam computed tomography CBCT (Galileos, Sirona, Germany) and visualization of planar reconstructions confirmed that the bent apical region was hooked into the cortical border of the nasal floor (Fig. 2). This morphologic characteristic of the canine apex was suspected to be the primary reason that the effort to force the tooth to its functional position was unsuccessful. Next, the recipient site of tooth eruption was examined both clinically and with CBCT reconstructions. The results revealed bone incompetence at the alveolar ridge and a palatal mucosal defect.

Therefore, autotransplantation of the canine was not considered feasible. The last alternative treatment option suggested for this case was the endodontic treatment of the exposed canine, followed by apicoectomy of the bent apical portion, which was anchored to the cortex.

The decision was based on the assumption that the downward movement of the canine along its normal eruption path was hindered by the anchoring of the bent apical portion in the dense bone. This anchor would not allow tooth advancement, regardless of the amount of force applied.

The patient was informed and agreed to the treatment plan, and the operation was scheduled. The endodontic treatment was performed 18 months after exposure of the impacted canine.

The root canal was prepared and filled to the apical bend.

An apicoectomy followed, under local anesthesia, essentially to remove the hooked apical portion of the tooth from the compact floor of the nasal cavity (Fig. 3). An initial post-operative control follow-up was performed a week after the operation.

Normal healing was observed, and the patient was asked to return for another follow-up appointment after the orthodontic treatment. The first recall took place 14 months after the operation. The canine had positively reacted to the treatment. During the post-operative period, force was applied on the bracket, and the canine had descended to its functional occlusion position.

At the next recall, 20 months postoperatively, no 


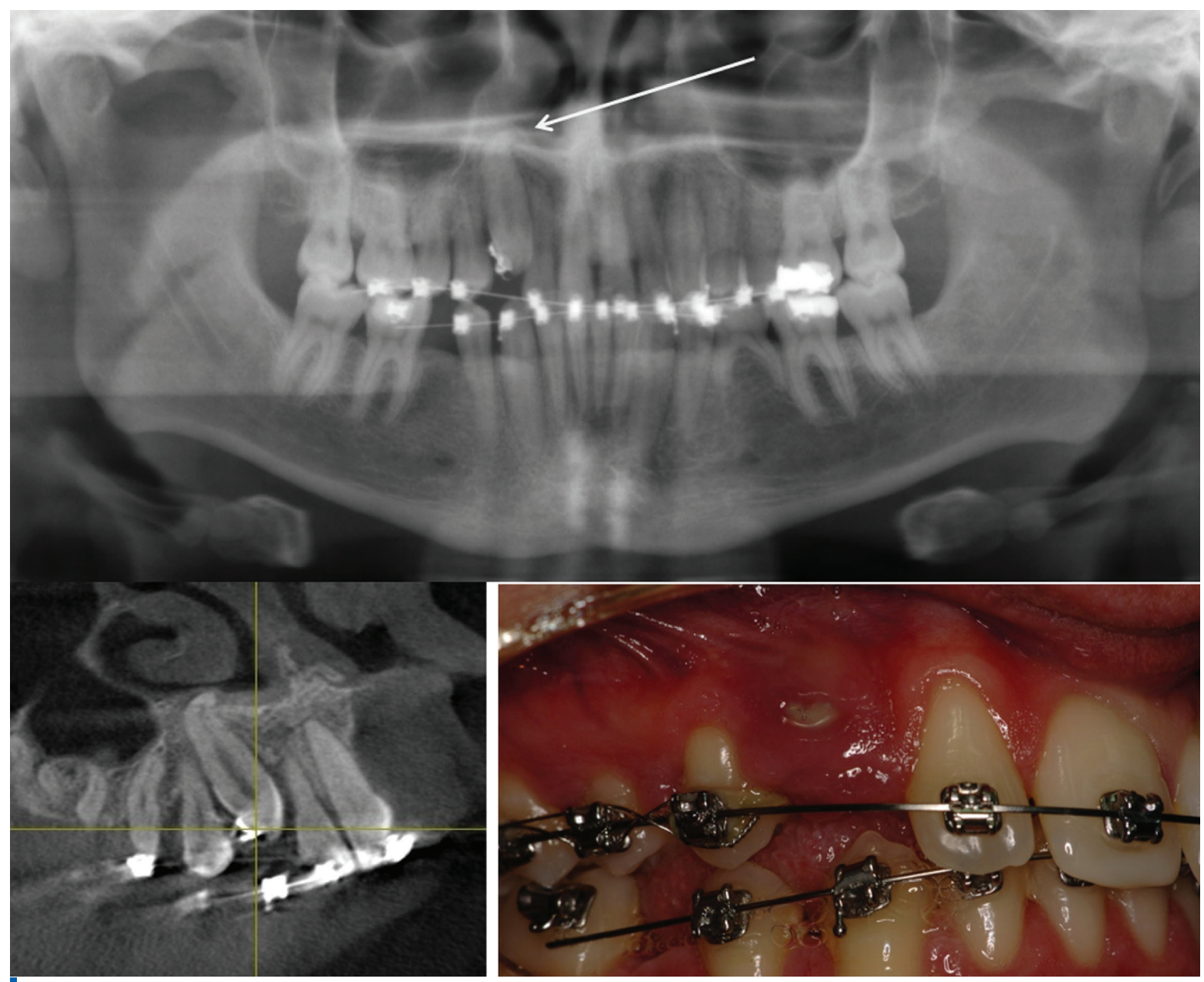

Figure 2. Panoramic radiograph and a CBCT reconstruction show the apical bend in the canine, which anchored it to the cortical bone of the nasal cavity floor. The canine could not descend to eruption, despite sufficient space and orthodontically-induced forces
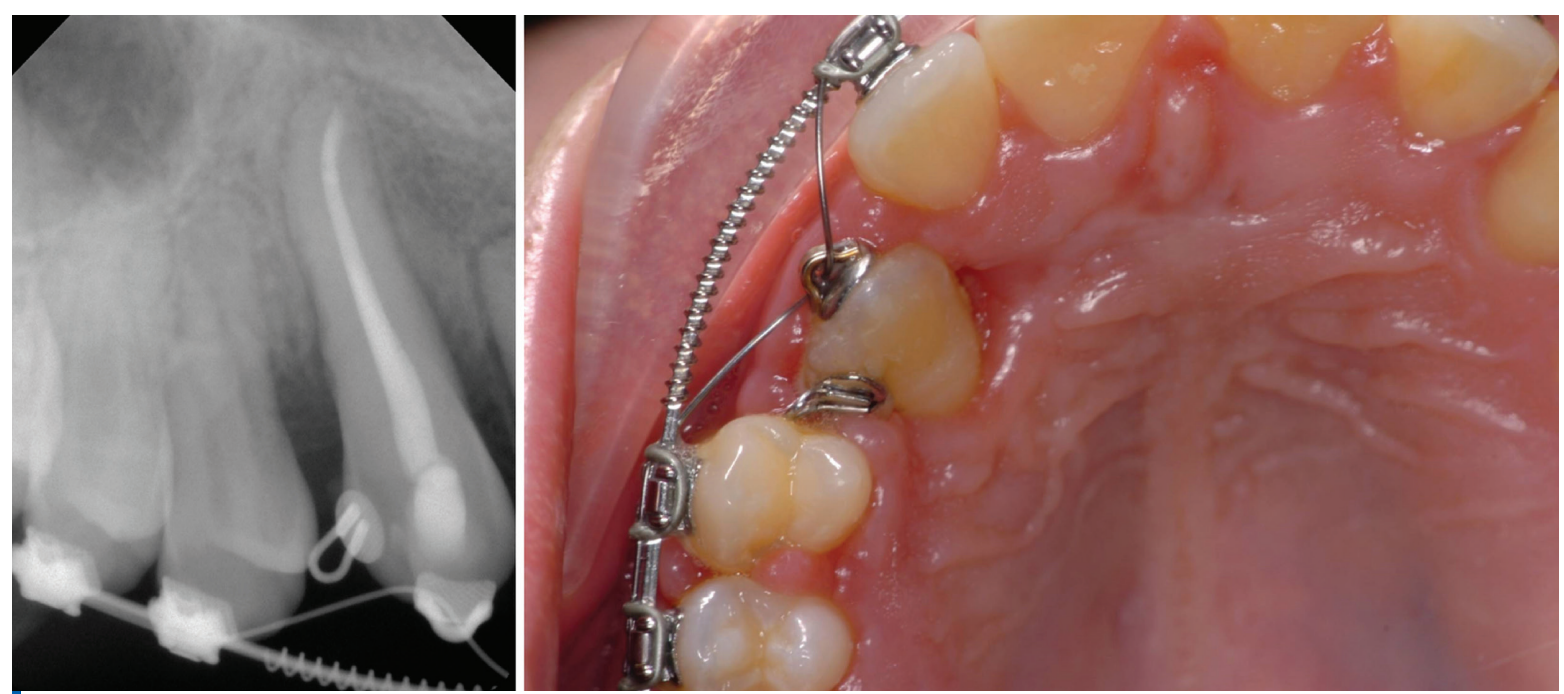

Figure 3. One week after apicoectomy of the apical bend, and after endodontic treatment

evidence of lesion or resorption was observed, and a third (last) follow-up appointment was scheduled (Fig. 4). At the last follow-up, 26 months postoperatively, a complete clinical and radiographic examination was performed. It included a panoramic radiograph and a CBCT scan, with lateral and cross-sectional reconstructions. The patient was symptom-free, and the right maxillary canine had maintained its functional position. There were no radiographic signs of periapical lesion or root resorption. The lamina dura was visible and the periodontal ligament space was normal. The radiographic examination showed a reduction of the alveolar bone height around the canine; this reduction was attributed to generalized periodontitis (Fig. 5). 

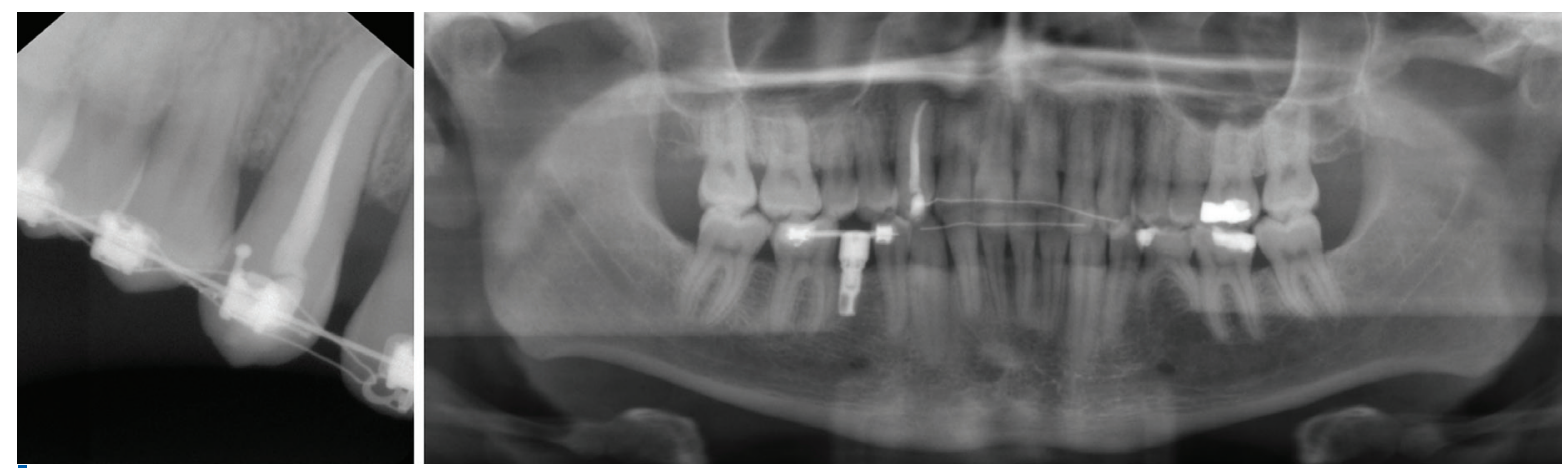

Figure 4. Postoperative follow-ups show successful results. The panormic radiograph taken 20 months postoperatively shows no further signs of a periapical lesion or root resorption
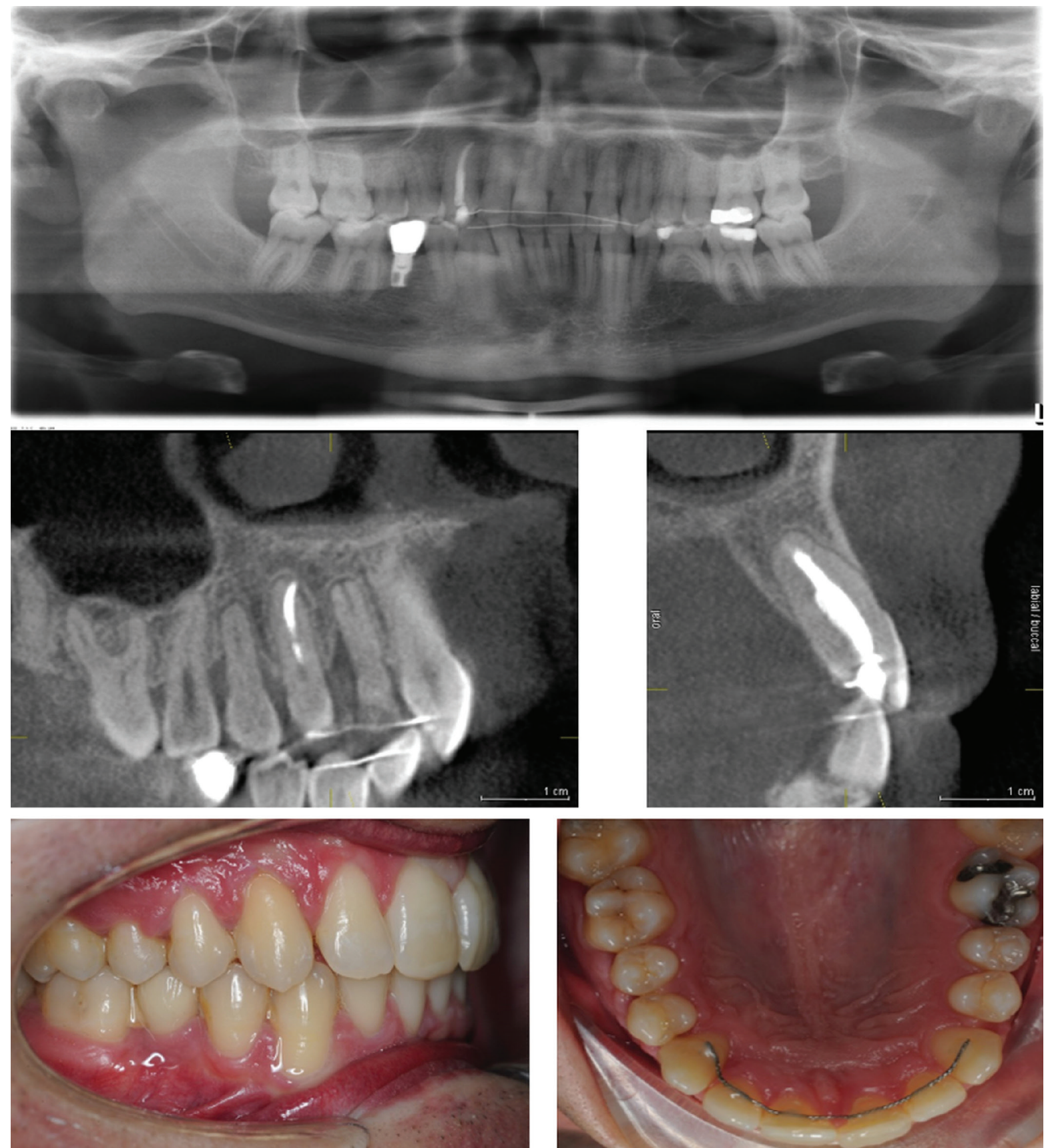

Figure 5. Two-year postoperative follow-up. Panoramic radiograph, CBCT lateral and cross-sectional reconstructions, and clinical photographs show the right maxillary canine in functional occlusion with no sign of periapical lesion or root resorption. The lamina dura is visible, and the periodontal ligament space is normal 


\section{Discussion}

Maxillary canine impaction is a well-documented dental condition. Many etiologic factors have been described in the literature. 7, 8,9 Impacted maxillary canines typically require a multidisciplinary approach. A correct diagnosis of the predisposing factors that cause tooth immobility is important for setting up a successful treatment plan ${ }^{10}$. Lack of space is a frequently reported cause of impaction, but palatally impacted maxillary canines have been associated with sufficient space in the dental arch. 5,6

This association was consistent with the clinical situation in the present case study; no crowding was observed at the recipient site, and the remaining space was favorable. With the first orthodontic treatment, the canine was exposed and a bracket was placed to force its eruption. Nevertheless, the impacted canine resisted the orthodontically-induced forces, which raised the suspicion of an ankylosis. However, ankylosis could be ruled out, based on results from mobility tests on the exposed canine crown and radiography (CBCT scan) of the periodontal ligament space around the root.

We next investigated a probable cause for the lack of mobility by examining reconstructions of the CBCT data in all planes. Finally, we found a morphological discrepancy of the canine that could explain the clinical situation; that is, we found an apical bend at the canine tip, which passed through the cortex of the nasal floor.

Autotransplantation was the first treatment alternative suggested, based on sufficient reports in the literature about successful rates in treating maxillary impacted canines; ${ }^{11,12}$ moreover, the recipient site had been opened orthodontically. However, an important factor in the prognosis of an autotransplanted tooth is the competence of the alveolar bone and the surrounding soft tissues in the recipient zone.

It is generally assumed that the periodontal ligament will not initiate osteogenesis in the absence of adjacent bone. Furthermore, wound closure with a gingival flap is a key factor in successful healing. ${ }^{13,14}$ In our case, the canine had previously been exposed and had commenced

\section{REFERENCES}

1. Aydin U, Yilmaz HH, Yildirim D. Incidence of canine impaction and transmigration in a patient population. Dentomaxillofac Radiol. 2004;33(3):164-169.

2. Grover PS, Lorton L. The incidence of unerupted permanent teeth and related clinical cases. Oral Surg Oral Med Oral Pathol. 1985;59(4):420-425.

3. Kramer RM, Williams AC. The incidence of impacted teeth. A survey at Harlem hospital. Oral Surg Oral Med Oral Pathol. 1970;29(2):237-241.

4. Celikoglu M, Kamak H, Oktay $H$. Investigation of transmigrated and impacted maxillary and mandibular canine teeth in an orthodontic patient population. J Oral Maxillofac Surg. 2010;68(5):1001-1006. descent towards the alveolar ridge before halting. However, we detected defects in the supporting bone tissue and the mucosa. Due to the lingual localization of these defects, we suspected that the condition of these tissues would deteriorate after the surgical removal of the canine.

Moreover, because these types of bony defects do not close optimally, ${ }^{15}$ they may potentially persist as periodontal defects after transplantation. Thus, the prognosis of an autotransplanted tooth was considered unfavorable.

The key to successful treatment for this patient was a careful assessment of the clinical situation, combined with early identification of the true cause of immobility (i.e., the dilaceration of the canine root apex, which anchored the canine to the cortical bone of the nasal floor).

The only alternative treatment option would have been an extraction of the immobile canine. Instead, we hypothesized that the hooked apical root tip had provided resistance to guided eruption. Therefore, we performed an apicoectomy of the anchored portion, after endodontically treating the exposed canine.

The follow-up examination performed 14 months postoperatively supported our hypothesis, because removal of the hindering factor allowed the canine to react positively, by promptly descending to its functional position with guided eruption.

A second follow-up examination was performed 26 months postoperatively. The treatment outcome was stable, as shown by the absence of mobility, resorption, periapical lesion, or recurrence of impaction.

\section{Conclusion}

An apical dilaceration through the cortical bone may immobilize an impacted canine. After treating the tooth endodontically, an apicoectomy of the bent tip can facilitate canine exposure and eruption, with a favorable prognosis.

\section{Acknowledgments}

The authors declare no conflict of interest related to this study. There are no conflicts of interest and no financial interests to be disclosed.

5. Chapokas AR, Almas K, Schincaglia GP. The impacted maxillary canine: a proposed classification for surgical exposure. Oral Surg Oral Med Oral Pathol Oral Radiol. 2012;113(2):222-228. 6. Jacoby $\mathrm{H}$. The etiology of maxillary canine impactions. Am J Orthod. 1983;84(2):125-132.

7. Jacobs SG. The impacted maxillary canine. Further observations on aetiology, radiographic localization, prevention/interception of impaction, and when to suspect impaction. Aust Dent J. 1996;41(5):310-316.

8. Richardson $G$, Russell KA. A review of impacted permanent maxillary cuspids--diagnosis and prevention. J Can Dent Assoc. 2000;66(9):497-501.

9. Power SM, Short MB. An investigation into the response 
of palatally displaced canines to the removal of deciduous canines and an assessment of factors contributing to favourable eruption. Br J Orthod. 1993;20(3):215-223.

10. Bishara SE. Impacted maxillary canines: a review. Am J Orthod Dentofacial Orthop. 1992;101(2):159-171. Review.

11. Thomas S, Turner SR, Sandy JR. Autotransplantation of teeth: is there a role? $\mathrm{Br} J$ Orthod. 1998;25(4):275-282. Review.

12. Lundberg $T$, Isaksson S. A clinical follow-up study of 278 autotransplanted teeth. Br J Oral Maxillofac Surg. 1996;34(2):181-185.
13. Tsukiboshi M, Andreasen JO. Autotransplantation of teeth 2001 Quintessence Pub. Co. Tsukiboshi M, Andreasen J0, Asai L, Bakland LK, Wilson TG Jr. Autotransplantation of teeth. 1st ed. Carol Stream, Illinois: Quintessence Publishing Co Inc; 2001. (p. 192).

14. Tsukiboshi M. Autotransplantation of teeth: requirements for predictable success. Dent Traumatol. 2002;18(4):157-180.

15. Collett T. Long-term follow up of clinical outcome of orthodontic tooth movement following extraction of previously auto-transplanted maxillary canines and bone grafting: a case report. Aust Orthod J. 2011;27(2):176-180.

\title{
Constantinus POLITIS \\ MD, DDS, PhD, Head
}

Department of Oral and Maxillofacial Surgery, Leuven University Hospitals, Department of Imaging and Pathology, KULeuven, Belgium

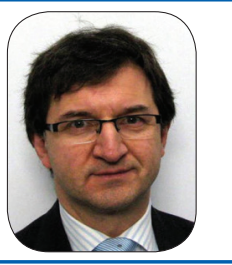

Dr. Constantinus Politis is Oral and Maxillo-Facial Surgeon. He is currently Professor and Chairperson of the Department of Oral and Maxillofacial Surgery at Leuven University, KULeuven, Belgium. He is invited Lecturer at the EHSAL in Brussels. He graduated at the Catholic University of Leuven in medicine (MD, summa cum laude), in dentistry (DDS, magna cum laude). He specialized in oral and maxillofacial surgery at the Catholic University of Leuven. Postgraduate training was additionally followed in Arnhem (Stoelinga), Aachen (Koberg), Copenhagen (Pindborg), Göteborg (Bränemark) and San Francisco (Marx). He also holds a master degree in management (MM) from the Applied Economic Scienes at the University of Hasselt and a master degree in Hospital Management (MHM) from the Catholic University of Leuven. He became a recognition as medical specialist in management of health care data and is now member of the National Council of Hospital Facilities. He is Secretary General of the Professional Union of Belgian Oral and Maxillofacial Surgeons.

$\mathrm{He}$ is acknowledged trainer of OMFS trainees. He defended his doctor's thesis on the subject of complications of orthognathic surgery (PhD).

His professional field of intrest is in orthognathic and orthodontic surgery and trigeminal nerve dysfunction. Clinical research projects include prevention and repair of iatrogenic trigeminal nerve injury; transplantation of teeth and orthognathic surgery.

\section{orvestions}

\section{Impacted canines are most frequently found:}

$\square$ a. In the palate;

$\square$ b. Buccaly in the maxilla;

c. Midcrestal in the maxilla;

d. In the mandible.

\section{Buccal displacement of maxillary canines is mostly caused by:}

$\square$ a. An insufficient dental arch length;

b. An odontoma;

c. Compact bone;

$\square$ d. A PTHR1 gene mutation.

\author{
Following syndrome is not associated with eruption disorders of teeth: \\ $\square$ a. GAPO-syndrome; \\ $\square$ b. Gardner syndrome; \\ $\square$ c. Dysostosis cleidocranialis; \\ $\square$ d. Gorlin-Gotz syndrome.
}

\section{Following sign is not a landmark sign of ankylosis after a heterotopic transplanta- tion of an impacted canine:}

$\square$ a. Pulp obliteration;

b. Loss of lamina dura;

c. Failure to respond to orthodontic forces;

$\square$ d. Replacement resorption. 